\title{
Summarizing Procedures in Concurrent Programs
}

\author{
Shaz Qadeer Sriram K. Rajamani Jakob Rehof \\ Microsoft Research \\ One Microsoft Way \\ Redmond, WA 98052
}

\begin{abstract}
The ability to summarize procedures is fundamental to building scalable interprocedural analyses. For sequential programs, procedure summarization is well-understood and used routinely in a variety of compiler optimizations and software defect-detection tools. However, the benefit of summarization is not available to multithreaded programs, for which a clear notion of summaries has so far remained unarticulated in the research literature.
\end{abstract}

In this paper, we present an intuitive and novel notion of procedure summaries for multithreaded programs. We also present a model checking algorithm for these programs that uses procedure summarization as an essential component. Our algorithm can also be viewed as a precise interprocedural dataflow analysis for multithreaded programs. Our method for procedure summarization is based on the insight that in well-synchronized programs, any computation of a thread can be viewed as a sequence of transactions, each of which appears to execute atomically to other threads. We summarize within each transaction; the summary of a procedure comprises the summaries of all transactions within the procedure. We leverage the theory of reduction [17] to infer boundaries of these transactions.

The procedure summaries computed by our algorithm allow reuse of analysis results across different call sites in a multithreaded program, a benefit that has hitherto been available only to sequential programs. Although our algorithm is not guaranteed to terminate on multithreaded programs that use recursion (reachability analysis for multithreaded programs with recursive procedures is undecidable [18]), there is a large class of programs for which our algorithm does terminate. We give a formal characterization of this class, which includes programs that use shared variables, synchronization, and recursion.

Categories and Subject Descriptors: D.1.3: Concurrent programming, parallel programming; D.2.4: Software/program verification

General Terms: Reliability, security, languages, verification

Keywords: Concurrent programs, pushdown systems, model checking, interprocedural dataflow analysis, procedure summaries, transactions, reduction

Permission to make digital or hard copies of all or part of this work for personal or classroom use is granted without fee provided that copies are not made or distributed for profit or commercial advantage and that copies bear this notice and the full citation on the first page. To copy otherwise, to republish, to post on servers or to redistribute

to lists, requires prior specific permission and/or a fee.

POPL'04, January 14-16, 2004, Venice, Italy.

Copyright 2004 ACM 1-58113-729-X/04/0001 ...\$5.00

\section{Introduction}

Sequential programs with finite-domain variables and recursive procedures are infinite-state systems due to unbounded stack depth. In spite of the potentially infinite state space of these programs, assertion checking is decidable for them. A common technique for analyzing such programs is CFL reachability [21, 19] (or equivalently, pushdown model checking $[22,10])$, where the key idea is to build procedure summaries. The summary of a procedure $P$ contains the state pair $\left(s, s^{\prime}\right)$ if in state $s$, there is an invocation of $P$ that yields the state $s^{\prime}$ on termination. Summaries enable reuse-if $P$ is called from two different places with the same state $s$, the work done in analyzing the first call is reused for the second. This reuse is the key to scalability of interprocedural analyses. Additionally, summarization avoids direct representation of the call stack, and guarantees termination of the analysis even if the program has recursion.

Assertion checking for multithreaded programs with finite-domain variables and recursive procedures is undecidable [18]. Most approaches to the analysis of such programs either restrict the interaction between synchronization and procedure calls $[1,9,6]$, or perform dataflow-style overapproximations [20], or use abstractions to capture the behavior of each thread $[4,12]$. In this paper, we take a radically different approach to solve this problem. We perform model checking directly on the multithreaded program. However, in order to gain reuse and scalability in our analysis, we present a novel method to compute procedure summaries even in the presence of multiple threads. Since the assertion checking problem is undecidable for multithreaded programs with procedures, we cannot guarantee termination of our algorithm on all cases. However, our algorithm terminates for a large class of multithreaded programs that includes programs with shared variables, synchronization, and recursion.

Our model checking algorithm for multithreaded programs has two levels. The first level performs reachability analysis and maintains an explicit stack for each thread. The second level computes a summary for each procedure. During the reachability analysis at the first level, whenever a thread makes a procedure call, we invoke the second level to compute a summary for the procedure. This summary is returned to the first level, which uses it to continue the reachability analysis.

The most crucial aspect of this algorithm is the notion of procedure summaries in multithreaded programs. A straightforward generalization of a (sequential) procedure summary to the case of multithreaded programs could attempt to accumulate all state pairs $\left(s, s^{\prime}\right)$ obtained by invoking this procedure in any thread. But this simple- 
minded extension is not that meaningful, since the resulting state $s$ for an invocation of a procedure $P$ in a thread might reflect updates by interleaved actions of concurrently executing threads. Clearly, these interleaved actions may depend on the local states of the other threads. Thus, if $\left(s, s^{\prime}\right)$ is an element of such a summary, and the procedure $P$ is invoked again by some thread in state $s$, there is no guarantee that the invoking thread will be in state $s^{\prime}$ on completing execution of $P$.

We present a robust and intuitive notion of procedure summaries for multithreaded programs. This notion is based on the insight that in well-synchronized programs, any computation of a thread can be viewed as a sequence of transactions, each of which appears to execute atomically to other threads. While analyzing the execution of a transaction by a thread, interleavings with other threads need not be considered. Our key idea is to summarize procedures within such transactions. Two main technical difficulties arise while performing transaction-based summarization of procedures:

- Transaction boundaries may not coincide with procedure boundaries. A transaction may begin in a procedure foo and end half-way inside a procedure bar called from foo. Conversely, a transaction may begin in a procedure foo and continue even after foo returns. One way to summarize such transactions is to have a stack frame as part of the state in each summary. However, this solution not only complicates the algorithm but also makes the summaries unbounded even if all state variables have a finite domain. Our summaries do not contain stack frames. If a transaction begins in one procedure context and ends in another procedure context, we break up the summary into smaller sub-summaries each within the context of a single procedure. Thus, our model checking algorithm uses a combination of two representations-states with stacks and summaries without stacks.

- A procedure can be called from different phases of a transaction - the pre-commit phase or the post-commit phase. We need to summarize the procedure differently depending on the phase of the transaction at the call site. We solve this problem by instrumenting the source program with a boolean variable representing the transaction phase, thus making the transaction phase part of the summaries.

We present a formal characterization of a class of multithreaded programs on which our summarization-based model checking algorithm is guaranteed to terminate. We show that if every call to a recursive procedure is contained entirely within a transaction, our algorithm will terminate with the correct answer.

To detect transactions in multithreaded programs, we leverage the theory of reduction [17]. Reduction views a transaction as a sequence of actions $a_{1}, \ldots, a_{m}, x, b_{1}, \ldots, b_{n}$ such that each $a_{i}$ is a right mover and each $b_{i}$ is a left mover. A right mover is an action that commutes to the right of every action by another thread; a left mover is an action that commutes to the left of every action by another thread. Thus, to detect transactions we need to detect right and left movers. In this paper, we abstract away from the problem of detecting movers and instead focus on the algorithm for summarization assuming that right and left movers are provided by a separate analysis $[15,14,13]$.

To summarize (!), we present a novel two-level model checking algorithm for multithreaded programs with (recursive) procedures. This algorithm uses transaction-based procedure summarization as a core component. We present a formal characterization of a class of programs on which our algorithm is guaranteed to terminate.

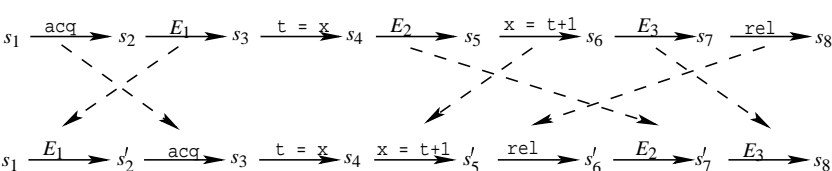

Figure 1. Transaction

This class includes a number of realistic programs that use shared variables, synchronization, and procedures. In recent years, flowsensitive and context-sensitive analyses have been used to check finite state properties of sequential programs in a scalable way using summarization as an essential component $[3,5]$. Our work is a first step in bringing these analyses to bear on multithreaded programs as well.

\section{Overview}

We illustrate our ideas using examples that use mutexes to protect accesses to shared variables. We first make some general observations about such programs.

- The action acquire (m), where $m$ is a mutex, is a right mover. Once it happens, there is no enabled action of another thread that may access $\mathrm{m}$. Hence, this action can be commuted to the right of any action of another thread.

- The action release (m) is a left mover. At a point when it is enabled but has not happened, there is no enabled action of another thread that may access m. Hence, this action can be commuted to the left of any action of another thread.

- An action that accesses only local variables is both a left mover and a right mover, since this action can be commuted both to the left and the right of actions by the other threads.

- An action that accesses a shared variable is both a left mover and right mover, as long as all threads acquire the same mutex before accessing that variable.

A transaction is a sequence of right movers, followed by a single atomic action with no restrictions, followed by a sequence of left movers. A transaction can be in two states: pre-commit or postcommit. A transactions starts in the pre-commit state and stays in the pre-commit state as long as right movers are being executed. When the atomic action (with no restrictions) is executed, the transaction moves to the post-commit state. This atomic action is called the committing action. The transaction stays in the post-commit state as long as left movers are being executed until the transaction completes.

We illustrate a transaction with an example. Figure 1 shows an execution of a concurrent program. A thread performs the following sequence of four operations: (1) acquires a lock (the operation acq in the first execution trace), (2) reads a variable $\mathrm{x}$ protected by that lock into a local variable $t(t=x),(3)$ updates that variable $(x=t+1)$, and (4) releases the lock (rel). Suppose that the actions of this method are interleaved with arbitrary actions $E_{1}, E_{2}, E_{3}$ of other threads. We assume that the environment actions respect the locking discipline of accessing $\mathrm{x}$ only after acquiring the lock.

Since the acquire operation is a right mover, it is commuted to the right of the environment action $E_{1}$ without changing the final state $s_{3}$, even though the intermediate state changes from $s_{2}$ to $s_{2}^{\prime}$. The read operation is the committing action. The write and release operations are left movers and are commuted to the left of environment 


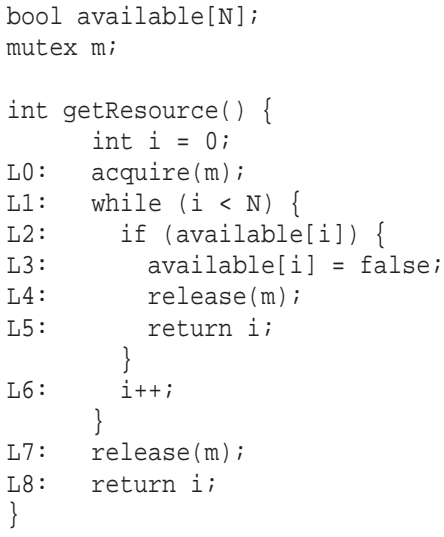

Figure 2. Resource allocator with coarse-grained locking

actions $E_{2}$ and $E_{3}$. Finally, after performing a series of commute operations, we get the execution at the bottom of the diagram in which the actions of the first thread happen without interruption from the environment. Note that in this execution the initial and final states are the same as before. Thus, the sequence of operations performed by the first thread constitute a transaction. This transaction is in the pre-commit state before the read of $\mathrm{x}$ and in the post-commit state afterwards.

We illustrate different aspects of our summarization-based model checking algorithm using four examples. The first example illustrates a simple case where the transaction boundary and the procedure boundary coincide. The second example illustrates summarization where a procedure body contains several transactions inside it. The third example illustrates that our algorithm terminates and validates the program assertions even in the presence of unbounded recursion. The fourth example illustrates our summarization technique when a procedure is called from different transactional contexts.

\section{Example 1}

Consider the resource allocation routine shown in Figure 2. There are $\mathrm{N}$ shared resources numbered $0, \ldots, \mathrm{N}-1$. The $j$-th entry in the global boolean array available is true iff the $j$-th resource is free to be allocated. A mutex m is used to protect accesses to available. The mutex $m$ has the value 0 when free, and 1 when locked. The body of getResource acquires the mutex $\mathrm{m}$, and then searches for the first available resource. If a free resource is found at index $i$, it sets available[i] to false and releases the mutex. If no free resource is found, then it releases the mutex. In both cases, it returns the final value of $i$. Thus, the returned value is the index of a free resource if one is available; otherwise, it is $\mathrm{N}$. There is a companion procedure freeResource for freeing an allocated resource, but we have not shown that in the figure. We assume that the multithreaded program consists of a number of threads that nondeterministically call getResource and freeResource.

Since acquire (m) is a right mover, release (m) is a left mover, and all other actions in getResource are both right and left movers, the entire procedure is contained in a single transaction. Suppose $\mathrm{N}=2$. We use $\left\langle a_{0}, a_{1}\right\rangle$ to denote the contents of available, where $a_{0}$ and $a_{1}$ respectively denote the values of available [0] and available[1]. The summary of getResource consists of a set of edges of the form $\left(p c, i, m,\left\langle a_{0}, a_{1}\right\rangle\right) \mapsto\left(p c^{\prime}, i^{\prime}, m^{\prime},\left\langle a_{0}^{\prime}, a_{1}^{\prime}\right\rangle\right)$,

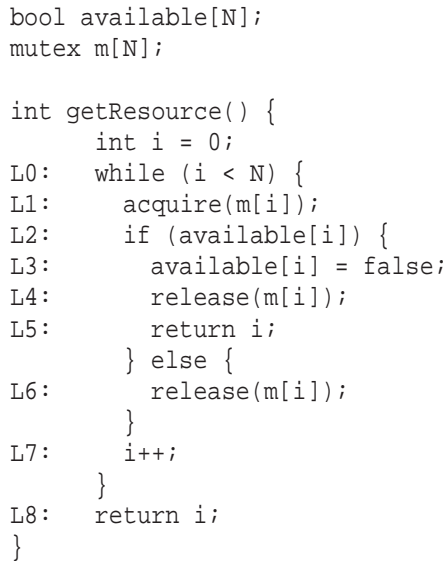

Figure 3. Resource allocator with fine-grained locking

where the tuple $\left(p c, i, m,\left\langle a_{0}, a_{1}\right\rangle\right)$ represents the values of the program counter and variables $i, m$, and available in the pre-store of the transaction and the tuple $\left(p c^{\prime}, i^{\prime}, m^{\prime},\left\langle a_{0}^{\prime}, a_{1}^{\prime}\right\rangle\right)$ denotes the corresponding values in the post-store of the transaction. The computed summary of getResource consists of the following edges:

$$
\begin{array}{lll}
(\mathrm{L} 0,0,0,\langle 0,0\rangle) & \mapsto & (\mathrm{L} 8,2,0,\langle 0,0\rangle) \\
(\mathrm{L} 0,0,0,\langle 0,1\rangle) & \mapsto & (\mathrm{L} 5,1,0,\langle 0,0\rangle) \\
(\mathrm{L} 0,0,0,\langle 1,0\rangle) & \mapsto & (\mathrm{L} 5,0,0,\langle 0,0\rangle) \\
(\mathrm{L} 0,0,0,\langle 1,1\rangle) & \mapsto & (\mathrm{L} 5,0,0,\langle 0,1\rangle)
\end{array}
$$

All edges in this summary begin at the label LO and terminate at one of the two labels - L 8 or L5-both of which are labels at which getResource returns. Thus, the summary matches the intuition that the procedure body is just one transaction. The first edge summarizes the case when no resource is allocated; the remaining three edges summarize the case when some resource is allocated. There is no edge beginning in a state with $\mathrm{m}=1$ since from such a state, the execution of the transaction blocks. Once this summary has been computed, if any thread calls getResource, the summary can be used to compute the state at the end of the transaction, without re-analyzing the body of getResource, thus providing reuse and scalability.

\section{Example 2}

Let us consider a modification to the resource allocator. The new program is shown in Figure 3. We have made the locking more fine-grained by using an array m of mutexes and protecting the $j$ th entry in available with the $j$-th entry in m. Now, the body of the procedure getResource is no longer contained entirely in a single transaction. In fact, there is one transaction corresponding to each iteration of the loop inside it. Again, suppose $\mathrm{N}=2$. Now, the summary contains edges of the form $\left(p c, i,\left\langle m_{0}, m_{1}\right\rangle,\left\langle a_{0}, a_{1}\right\rangle\right) \mapsto$ $\left(p c^{\prime}, i^{\prime},\left\langle m_{0}^{\prime}, m_{1}^{\prime}\right\rangle,\left\langle a_{0}^{\prime}, a_{1}^{\prime}\right\rangle\right)$, where $\left\langle m_{0}, m_{1}\right\rangle$ denotes the contents of $\mathrm{m}$ in the pre-store and $\left\langle m_{0}^{\prime}, m_{1}^{\prime}\right\rangle$ denotes the contents of $\mathrm{m}$ in the poststore. The computed summary of getResource consists of the following edges:

$$
\begin{array}{lll}
(\mathrm{L} 0,0,\langle 0,0\rangle,\langle 0,0\rangle) & \mapsto & (\mathrm{L} 1,1,\langle 0,0\rangle,\langle 0,0\rangle) \\
(\mathrm{L} 0,0,\langle 0,0\rangle,\langle 0,1\rangle) & \mapsto & (\mathrm{L} 1,1,\langle 0,0\rangle,\langle 0,1\rangle) \\
(\mathrm{L} 0,0,\langle 0,0\rangle,\langle 1,0\rangle) & \mapsto & (\mathrm{L} 5,0,\langle 0,0\rangle,\langle 0,0\rangle) \\
(\mathrm{L} 0,0,\langle 0,0\rangle,\langle 1,1\rangle) & \mapsto & (\mathrm{L} 5,0,\langle 0,0\rangle,\langle 0,1\rangle)
\end{array}
$$




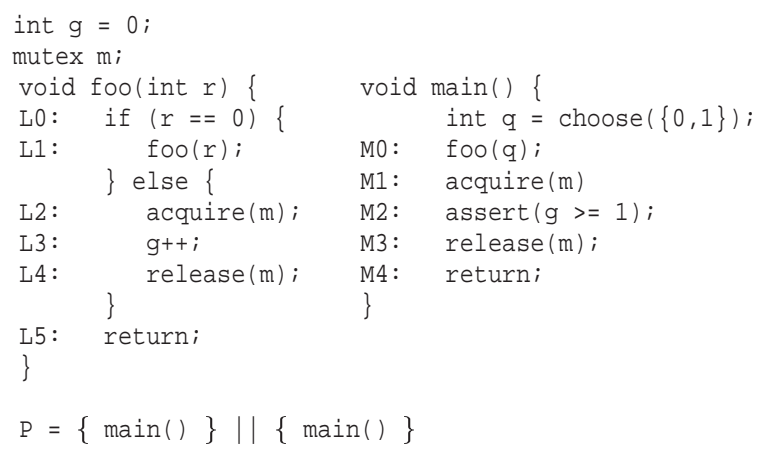

Figure 4. Summarization enables termination

$$
\begin{array}{lll}
(\mathrm{L} 1,1,\langle 0,0\rangle,\langle 0,0\rangle) & \mapsto & (\mathrm{L} 8,2,\langle 0,0\rangle,\langle 0,0\rangle) \\
(\mathrm{L} 1,1,\langle 0,0\rangle,\langle 0,1\rangle) & \mapsto & (\mathrm{L} 5,1,\langle 0,0\rangle,\langle 0,0\rangle) \\
(\mathrm{L} 1,1,\langle 0,0\rangle,\langle 1,0\rangle) & \mapsto & (\mathrm{L} 8,2,\langle 0,0\rangle,\langle 1,0\rangle) \\
(\mathrm{L} 1,1,\langle 0,0\rangle,\langle 1,1\rangle) & \mapsto & (\mathrm{L} 5,1,\langle 0,0\rangle,\langle 1,0\rangle)
\end{array}
$$

This summary contains three kinds of edges. The first two edges correspond to the transaction that starts at the beginning of the procedure, i.e., at label L 0 with $i=0$, goes through the loop once, and ends at L1 with $i=1$. The next two edges correspond to the transaction that again starts at the beginning of the procedure, but ends with the return at label $\mathrm{L} 5$ during the first iteration through the loop. The last four edges correspond to the transaction that starts in the middle of the procedure at label L1 with $i=1$, and returns either at label L5 or L8. Note that edges of the first and third kind did not exist in the summary of the previous version of getResource, where all edges went from entry to exit.

\section{Example 3}

The previous two examples illustrated summaries and the reuse afforded by them. In a number of programs, summaries enable our model checking algorithm to terminate where the naive model checking algorithm without summaries will not terminate. Consider the example in Figure 4. The program P consists of two threads, each of which starts execution by calling the main procedure. The main procedure has a local variable $q$ which is initialized nondeterministically. Then main calls foo with $q$ as the actual parameter. The procedure foo has an infinite recursion if the parameter $r$ is 0 . Otherwise, it increments global $g$ and returns. After returning from foo, the main procedure asserts that $(g>=1)$. All accesses to the shared global $g$ are protected by a mutex $\mathrm{m}$. The initial value of $g$ is 0 .

The stack can grow without bound due to the recursion in procedure foo. Hence, naive model checking does not terminate on this example. However, the body of foo consists of one transaction, since all action sequences in foo consist of a sequence of right movers followed by a sequence of left movers. A summary edge for foo is of the form $(p c, r, m, g) \mapsto\left(p c^{\prime}, r^{\prime}, m^{\prime}, g^{\prime}\right)$, whose meaning is similar to that of a summary edge in the previous examples. The summary for foo consists of the following edges:

$$
\begin{array}{lll}
(\mathrm{L} 0,1,0,0) & \mapsto \quad(\mathrm{L} 5,1,0,1) \\
(\mathrm{L} 0,1,0,1) & \mapsto \quad(\mathrm{L} 5,1,0,2)
\end{array}
$$

There is no edge beginning in a state with $r=0$ since from such a state, the execution of the transaction never terminates. Using summaries, we avoid reasoning about the stack explicitly inside foo and also avoid exploring the unbounded recursion in foo.

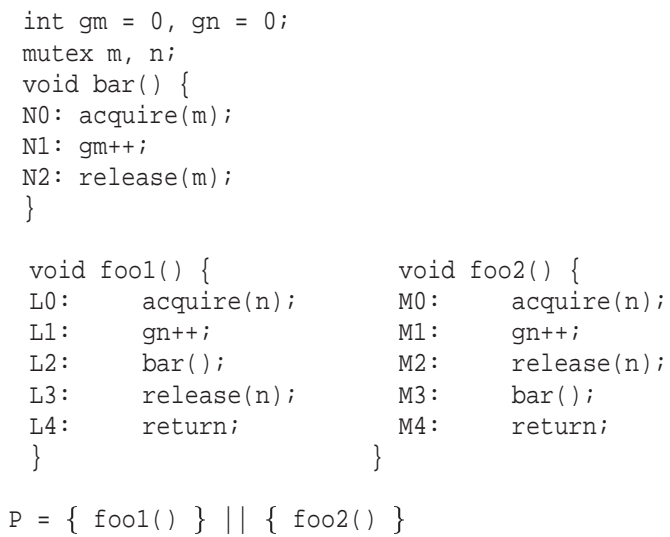

Figure 5. Summarization from different transactional contexts

The body of main has two transactions. The first transaction begins at label M0 and ends at label M1, consisting of essentially the call to foo. The second transaction begins at label M1 and ends at label M4. A summary edge for main has the form $(p c, q, m, g) \mapsto$ $\left(p c, q^{\prime}, m^{\prime}, g^{\prime}\right)$. The summary for main consists of the following edges:

$$
\begin{array}{lll}
(\mathrm{M} 0,1,0,0) & \mapsto & (\mathrm{M} 1,1,0,1) \\
(\mathrm{M} 0,1,0,1) & \mapsto & (\mathrm{M} 1,1,0,2) \\
& & \\
(\mathrm{M} 1,1,0,1) & \mapsto & (\mathrm{M} 4,1,0,1) \\
(\mathrm{M} 1,1,0,2) & \mapsto & (\mathrm{M} 4,1,0,2)
\end{array}
$$

Using these summaries for procedures foo and main, our model checking algorithm is able to terminate and correctly conclude that $P$ is free of assertion violations. The algorithm begins with an empty stack for each thread. When a thread calls main, since the body of main is not contained within one transaction, the algorithm pushes a frame for main on the stack of the calling thread. However, when a thread calls foo, no frame corresponding to foo is pushed since the entire body of $f \circ o$ is contained within a transaction. Instead, foo is summarized and its summary is used to make progress in the model checking.

\section{Example 4}

Consider the example in Figure 5. Here, two shared variables gm and $g n$ are protected by mutexes $m$ and $n$ respectively. Procedure bar accesses the variable gm, and is called from two different procedures foo 1 and foo2. In foo1, the procedure bar is called from the pre-commit state of the transaction, since no mutexes are released prior to calling bar. In foo2, the procedure bar is called from the post-commit state of the transaction, since mutex $n$ is released prior to calling bar. The summary for bar needs to distinguish these two calling contexts. In the case of the call from fool, the entire body of foo1 including the call to bar () is part of the same transaction. In the case of the call from foo2, there are two transactions, one from label M0 to M3, and another from label M3 to M4. We distinguish these two by instrumenting the semantics of the program with an extra bit of information that records the phase of the transaction. Then, each summary edge provides the pre and post values not only of program variables but also of the transaction phase. More details are given Section 4. 


\section{Multithreaded programs}

The store of a multithreaded program is partitioned into the global store Global and the local store Local of each thread. The set Local of local stores has a special store called wrong. The local store of a thread moves to wrong on failing an assertion and thereafter the failed thread does not make any other transitions.

\begin{tabular}{|c|c|c|c|c|}
\hline \multicolumn{5}{|c|}{ Domains } \\
\hline$t, u$ & $\in$ & Tid & $=$ & $\{1, \ldots, n\}$ \\
\hline$g$ & $\in$ & Global & & \\
\hline$l$ & $\in$ & Local & & \\
\hline$l s$ & $\in$ & Locals & $=$ & Tid $\rightarrow$ Local \\
\hline$f$ & $\epsilon$ & Frame & & \\
\hline$s$ & $\in$ & Stack & $=$ & Frame $^{*}$ \\
\hline ss & $\epsilon$ & Stacks & $=$ & Tid $\rightarrow$ Stack \\
\hline & & State & $=$ & Global $\times$ Locals $\times$ Stacks \\
\hline
\end{tabular}

A multithreaded program $\left(g_{0}, l s_{0}, T, T^{+}, T^{-}\right)$consists of five components. $g_{0}$ is the initial value of the global store. $l s_{0}$ maps each thread id $t \in \operatorname{Tid}$ to the initial local store $l s_{0}(t)$ of thread $t$. We model the behavior of the individual threads using three relations:

$$
\begin{array}{rll}
T & \subseteq & \text { Tid } \times(\text { Global } \times \text { Local }) \times(\text { Global } \times \text { Local }) \\
T^{+} & \subseteq & \text { Tid } \times \text { Local } \times(\text { Local } \times \text { Frame }) \\
T^{-} & \subseteq & \text { Tid } \times(\text { Local } \times \text { Frame }) \times \text { Local }
\end{array}
$$

The relation $T$ models thread steps that do not manipulate the stack. The relation $T\left(t, g, l, g^{\prime}, l^{\prime}\right)$ holds if thread $t$ can take a step from a state with global store $g$ and local store $l$, yielding (possibly modified) stores $g^{\prime}$ and $l^{\prime}$. The stack is not accessed or updated during this step. The relation $T^{+}\left(t, l, l^{\prime}, f\right)$ models steps of thread $t$ that push a frame onto the stack. This step does not access the global store, is enabled when the local store is $l$, updates the local store to $l^{\prime}$, and pushes the frame $f$ onto the stack. Similarly, the relation $T^{-}\left(t, l, f, l^{\prime}\right)$ models steps of thread $t$ that pop a frame from the stack. This step also does not access the global store, is enabled when the local store is $l$ and the frame at the top of the stack is $f$, updates the local store to $l^{\prime}$, and pops the frame $f$ from the stack.

The program starts execution from the state $\left(g_{0}, l s_{0}, s s_{0}\right)$ where $s s_{0}(t)=\varepsilon$ for all $t \in$ Tid. At each step, any thread may make a transition. The transition relation $\rightarrow_{t} \subseteq$ State $\times$ State of thread $t$ is defined below. For any function $h$ from $A$ to $B, a \in A$ and $b \in B$, we write $h[a:=b]$ to denote a new function such that $h[a:=b](x)$ evaluates to $h(x)$ if $x \neq a$, and to $b$ if $x=a$.

Transition relation $\rightarrow_{t}$

$$
\begin{gathered}
\frac{T\left(t, g, l s(t), g^{\prime}, l^{\prime}\right)}{(g, l s, s s) \rightarrow_{t}\left(g^{\prime}, l s\left[t:=l^{\prime}\right], s s\right)} \\
\frac{T^{+}\left(t, l s(t), l^{\prime}, f\right)}{(g, l s, s s) \rightarrow_{t}\left(g, l s\left[t:=l^{\prime}\right], s s[t:=s s(t) . f]\right)} \\
\frac{s s(t)=s . f \quad T^{-}\left(t, l s(t), f, l^{\prime}\right)}{(g, l s, s s) \rightarrow_{t}\left(g, l s\left[t:=l^{\prime}\right], s s[t:=s]\right)}
\end{gathered}
$$

The transition relation $\rightarrow \subseteq$ State $\times$ State of the program is the disjunction of the transition relations of the various threads.

$$
\rightarrow \quad=\quad \exists t . \rightarrow_{t}
$$

\section{Model checking with reduction}

Transactions occur in multithreaded programs because of the presence of right and left movers. Inferring which actions of a program are right and left movers is a problem that is important but orthogonal to the contribution of this paper. In this section, we assume that right and left movers are available to us as the result of a previous analysis (see, e.g. $[15,14])$. We use this information to derive a model checking algorithm that uses transactions but does not perform any summarization of procedures. We will use the intuition developed in this section to derive a second model checking algorithm in Section 5 that performs procedure summarization as well.

Let $R M, L M \subset T$ be subsets of the transition relation $T$ with the following two properties for all $t \neq u$ :

1. If $R M\left(t, g_{1}, l_{1}, g_{2}, l_{2}\right)$ and $T\left(u, g_{2}, l_{3}, g_{3}, l_{4}\right)$, there is $g_{4}$ such that $T\left(u, g_{1}, l_{3}, g_{4}, l_{4}\right)$ and $R M\left(t, g_{4}, l_{1}, g_{3}, l_{2}\right)$. Further, $R M\left(u, g_{2}, l_{3}, g_{3}, l_{4}\right)$ iff $R M\left(u, g_{1}, l_{3}, g_{4}, l_{4}\right)$, and $L M\left(u, g_{2}, l_{3}, g_{3}, l_{4}\right)$ iff $\operatorname{LM}\left(u, g_{1}, l_{3}, g_{4}, l_{4}\right)$.

2. If $T\left(u, g_{1}, l_{1}, g_{2}, l_{2}\right)$ and $\operatorname{LM}\left(t, g_{2}, l_{3}, g_{3}, l_{4}\right)$, there is $g_{4}$ such that $\operatorname{LM}\left(t, g_{1}, l_{3}, g_{4}, l_{4}\right)$ and $T\left(u, g_{4}, l_{1}, g_{3}, l_{2}\right)$. Further, $R M\left(u, g_{1}, l_{1}, g_{2}, l_{2}\right)$ iff $R M\left(u, g_{4}, l_{1}, g_{3}, l_{2}\right), \quad$ and $\operatorname{LM}\left(u, g_{1}, l_{1}, g_{2}, l_{2}\right)$ iff $\operatorname{LM}\left(u, g_{4}, l_{1}, g_{3}, l_{2}\right)$.

The first property states that a right mover action in thread $t$ commutes to the right of an action of a different thread $u$. Moreover, the action by thread $u$ is a right mover (resp. left mover) before the commute operation iff it is a right mover (resp. left mover) after the commute operation. Similarly, the second property states the requirement on a left mover in thread $t$. Our analysis is parameterized by the values of $R M$ and $L M$ and only requires that they satisfy these two properties. The larger the relations $R M$ and $L M$, the longer the transactions our analysis infers. Therefore, these relations should be as large as possible in practice.

As mentioned before, a transaction is a sequence of right movers followed by a single action followed by a sequence of left movers. In order to minimize the number of explored interleavings and to maximize reuse, we would like to infer transactions that are as long as possible. In order to implement this inference, we introduce in each thread a boolean local variable to keep track of the phase of that thread's transaction. Note that this instrumentation is done automatically by our analysis, and not by the programmer. The phase variable of thread $t$ is true if thread $t$ is in the right mover (or pre-commit) part of the transaction; otherwise the phase variable is false. We say that the transaction commits when the phase variable moves from true to false. The initial value of the phase variable for each thread is true.

$$
\begin{array}{rlll}
p, p^{\prime} & \in & \text { Boolean } & \\
\ell, \ell^{\prime} & \in \text { Local }^{\#} & = & \text { Local } \times \text { Boolean } \\
\ell s, \ell s^{\prime} & \in \text { Locals }^{\#}= & \text { Tid } \rightarrow \text { Local }^{\#}
\end{array}
$$

The initial value of the global store of the instrumented program remains $g_{0}$. The initial value of the local stores changes to $\ell s_{0}$, where $\ell s_{0}(t)=\left\langle l s_{0}(t)\right.$, true $\rangle$ for all $t \in$ Tid. We instrument the transition relation $T, T^{+}$, and $T^{-}$to generate new transition relations $U, U^{+}$, and $U^{-}$that update the phase appropriately.

$$
\begin{aligned}
U & \subseteq \text { Tid } \times\left(\text { Global } \times \text { Local }^{\#}\right) \times\left(\text { Global } \times \text { Local }^{\#}\right) \\
U^{+} & \subseteq \text { Tid } \times \text { Local }^{\#} \times\left(\text { Local }^{\#} \times \text { Frame }\right) \\
U^{-} & \subseteq \text { Tid } \times\left(\text { Local }^{\#} \times \text { Frame }\right) \times \text { Local }^{\#}
\end{aligned}
$$


Ruleset 1: Model checking with reduction (INIT)

$$
\overline{\Sigma\left(g_{0}, \ell s_{0}, s s_{0}\right)}
$$

(STEP)

$$
\begin{gathered}
\forall u \neq t . \mathcal{N}(u, g, \ell s(u)) \\
\frac{\Sigma(g, l s, s s) \quad U\left(t, g, \ell s(t), g^{\prime}, \ell^{\prime}\right)}{\Sigma\left(g^{\prime}, \ell s\left[t:=\ell^{\prime}\right], s s\right)}
\end{gathered}
$$

(PUSH)

$$
\begin{gathered}
\forall u \neq t . \mathcal{N}(u, g, \ell s(u)) \\
\Sigma(g, \ell s, s s) \quad U^{+}\left(t, \ell s(t), \ell^{\prime}, f\right) \\
\hline \Sigma\left(g, \ell s\left[t:=\ell^{\prime}\right], s s[t:=s s(t) . f]\right)
\end{gathered}
$$

(POP)

$$
\begin{gathered}
\forall u \neq t . \mathcal{N}(u, g, \ell s(u)) \\
\frac{\Sigma(g, \ell s, s s) \quad U^{-}\left(t, \ell s(t), f, \ell^{\prime}\right) \quad s s(t)=s . f}{\Sigma\left(g, \ell s\left[t:=\ell^{\prime}\right], s s[t:=s]\right)}
\end{gathered}
$$

$$
\begin{aligned}
& U\left(t, g,\langle l, p\rangle, g^{\prime},\left\langle l^{\prime}, p^{\prime}\right\rangle\right) \stackrel{\text { def }}{=} \\
& \wedge T\left(t, g, l, g^{\prime}, l^{\prime}\right) \\
& \wedge \quad p^{\prime}=\left(R M\left(t, g, l, g^{\prime}, l^{\prime}\right) \wedge\left(p \vee \neg L M\left(t, g, l, g^{\prime}, l^{\prime}\right)\right)\right) \\
& U^{+}\left(t,\langle l, p\rangle,\left\langle l^{\prime}, p^{\prime}\right\rangle, f\right) \stackrel{\text { def }}{=} \\
& \wedge T^{+}\left(t, l, l^{\prime}, f\right) \\
& \wedge \quad p^{\prime}=p \\
& U^{-}\left(t,\langle l, p\rangle, f,\left\langle l^{\prime}, p^{\prime}\right\rangle\right) \stackrel{\text { def }}{=} \\
& \wedge T^{-}\left(t, l, f, l^{\prime}\right) \\
& \wedge \quad p^{\prime}=p
\end{aligned}
$$

In the definition of $U$, the relation between $p^{\prime}$ and $p$ reflects the intuition that if $p$ is true, then $p^{\prime}$ continues to be true as long as it executes right mover actions. The phase changes to false as soon as the thread executes an action that is not a right mover. Thereafter, it remains false as long as the thread executes left movers. Then, it becomes true again as soon as the thread executes an action that is a right mover and not a left mover.

For each thread $t$, we define three sets:

$$
\mathcal{R}(t), \mathcal{L}(t), \mathcal{N}(t) \subseteq \text { Global } \times \text { Local }^{\#}
$$

These sets respectively define when a thread is executing in the right mover part of a transaction, the left mover part of a transaction, and outside any transaction. For example, in the first execution of Figure 1, let $t$ be the identifier of the thread executing the transaction. Then, the states $\left\{s_{2}, s_{3}\right\} \in \mathcal{R}(t),\left\{s_{4}, s_{5}, s_{6}, s_{7}\right\} \in \mathcal{L}(t)$, and $\left\{s_{1}, s_{8}\right\} \in \mathcal{N}(t)$. These three sets can be any partition of $\left(\right.$ Global $\times$ Local $\left.^{\#}\right)$ satisfying the following two conditions:

$$
\begin{aligned}
& \text { C1. } \mathcal{R}(t) \subseteq\left\{(g,\langle l, p\rangle) \mid l \notin\left\{l s_{0}(t), \text { wrong }\right\} \wedge p\right\} . \\
& \text { C2. } \mathcal{L}(t) \subseteq\left\{\begin{array}{l|l}
(g,\langle l, p\rangle) \mid \begin{array}{l}
l \notin\left\{l s_{0}(t), \text { wrong }\right\} \wedge \neg p \wedge \\
\forall g^{\prime}, l^{\prime} . T\left(t, g, l, g^{\prime}, l^{\prime}\right) \\
\Rightarrow L M\left(t, g, l, g^{\prime}, l^{\prime}\right)
\end{array}
\end{array}\right\} .
\end{aligned}
$$

Condition C1 says that thread $t$ is in the right mover part of a transaction only if the local store of $t$ is neither its initial value nor wrong and the phase variable is true. Condition C2 says that thread $t$ is in the left mover part of a transaction only if the local store of $t$ is neither its initial value nor wrong, the phase variable is false, and all possible enabled transitions are left movers. Since
$(\mathcal{R}(t), \mathcal{L}(t), \mathcal{N}(t))$ is a partition of $\left(\right.$ Global $\times$ Local $\left.^{\#}\right)$, once $\mathcal{R}(t)$ and $\mathcal{L}(t)$ have been picked according to $\mathrm{C} 1$ and $\mathrm{C} 2$, the set $\mathcal{N}(t)$ is implicitly defined.

We write $\mathcal{R}(t, g, \ell)$ whenever $(g, \ell) \in \mathcal{R}(t), \mathcal{L}(t, g, \ell)$ whenever $(g, \ell) \in \mathcal{L}(t)$, and $\mathcal{N}(t, g, \ell)$ whenever $(g, \ell) \in \mathcal{N}(t)$. Finally, using the values of $\mathcal{N}(t)$ for all $t \in$ Tid, we model check the multithreaded program by computing the least fixpoint of the set of rules in Ruleset 1 . This model checking algorithm schedules a thread only when no other thread is executing inside a transaction.

Conditions $\mathrm{C} 1$ and $\mathrm{C} 2$ are not quite enough for the model checking algorithm to be sound. The reason is the following. If a transaction in thread $t$ commits but never finishes, the shared variables modified by this transaction become visible to other threads. However, the algorithm does not explore transitions of other threads from any state after the transaction commits. Therefore, we add a third condition $\mathrm{C} 3$ which states that every committed transaction must finish. In order to state this condition formally, we extend the transition relation $\rightarrow_{t}$ from Section 3 to the program store augmented with the phase variable in the natural way.

C3. Suppose $(g, \ell) \in \mathcal{L}(t), \Sigma(g, \ell s, s s)$, and $\ell s(t)=\ell$. Then, there is $g^{\prime}, \ell s^{\prime}$, and $s s^{\prime}$ such that $\left(g^{\prime}, \ell s^{\prime}(t)\right) \in \mathcal{N}(t)$ and $(g, \ell s, s s) \rightarrow_{t}^{*}$ $\left(g^{\prime}, \ell s^{\prime}, s s^{\prime}\right)$.

Our analysis is correct for any partition $(\mathcal{R}(t), \mathcal{L}(t), \mathcal{N}(t))$ of $\left(\right.$ Global $\times$ Local $\left.^{\#}\right)$ satisfying conditions $\mathrm{C} 1, \mathrm{C} 2$, and $\mathrm{C} 3$. The smaller the value of $\mathcal{N}(t)$, the larger the transactions inferred by the analysis. Therefore, an implementation of our analysis should pick a value for $\mathcal{N}(t)$ that is as small as possible. We can now state our soundness theorem for the model checking algorithm presented above.

THEOREM 1. Let $\left(g_{0}, \ell s_{0}, U, U^{+}, U^{-}\right)$be the instrumented multithreaded program. Let $\Sigma$ be the least fixpoint of the rules in Ruleset 1. Let the conditions C1, C2, and C3 be satisfied. If $\left(g_{0}, l s_{0}, s s_{0}\right) \rightarrow^{*}(g, l s, s s)$ and $l s(t)=$ wrong, then there is $\left(g^{\prime}, \ell s^{\prime}, s s^{\prime}\right)$ and $p$ such that $\Sigma\left(g^{\prime}, \ell s^{\prime}, s s^{\prime}\right)$ and $\ell s^{\prime}(t)=\langle$ wrong,$p\rangle$.

Proof (Sketch) Suppose $\left(g_{0}, l s_{0}, s s_{0}\right) \rightarrow^{*}(g, l s, s s)$ through some sequence of actions of various threads and $l s(t)=$ wrong. First, we extend this sequence to complete all committed but unfinished transactions using condition $\mathrm{C} 3$. Then, one by one, we commute each action in an uncommitted transaction to the right and drop it. Eventually, we will get an execution sequence $\sigma$ with only completed transactions and with the property that $\sigma$ goes wrong if the original sequence goes wrong. Therefore $\sigma$ goes wrong as well. In $\sigma$, the transactions of a thread could have interleaved actions of another thread. The order in which transactions commit is a total order on the transactions in $\sigma$. We denote this total order by $<$. We can transform $\sigma$ into an equivalent execution $\sigma^{\prime}$ (by appropriately rightcommuting right movers, and left-commuting left movers), such that $\sigma^{\prime}$ has the following properties: (1) for every thread $t$, no action of a different thread $t^{\prime}$ occurs in the middle of a transaction of thread $t$, (2) the transactions in $\sigma^{\prime}$ commit in the order $<$. From the properties of right and left movers, we get that $\sigma^{\prime}$ also goes wrong. Since $\sigma^{\prime}$ schedules each transaction to completion, the states along $\sigma^{\prime}$ will be explored by the rules in Ruleset 1 . A similar proof has been carried out with more detail in an earlier paper [15].

Although this algorithm is sound, it might not terminate if a thread calls a recursive procedure (even if all variables take values from a finite domain). In the next section, we use the concepts developed in this section to derive a model checking algorithm that uses proce- 
Ruleset 2: Level I-Reachability

(INIT)

$$
\overline{\Omega\left(g_{0}, \ell s_{0}, s s_{0}\right)}
$$

(STEP)

$$
\begin{gathered}
\Omega(g, \ell s, s s) \quad \forall u \neq t . \mathcal{N}(u, g, \ell s(u)) \\
\operatorname{Sum}\left(t, g, \ell s(t), g^{\prime}, \ell^{\prime}\right) \\
\hline \Omega\left(g^{\prime}, \ell s\left[t:=\ell^{\prime}\right], s s, p s\left[t:=p^{\prime}\right]\right)
\end{gathered}
$$

(PUSH)

$$
\begin{gathered}
\Omega(g, \ell s, s s) \quad \forall u \neq t . \mathcal{N}(u, g, \ell s(u)) \\
\operatorname{Sum}^{+}\left(t, g, \ell s(t), g^{\prime}, \ell^{\prime}, f\right) \\
\hline \Omega\left(g^{\prime}, \ell s\left[t:=\ell^{\prime}\right], s s[t:=s s(t) . f]\right)
\end{gathered}
$$

(POP)

$$
\begin{array}{cc}
\Omega(g, l s, s s) & \forall u \neq t . \mathcal{N}(u, g, \ell s(u)) \\
s s(t)=s . f & \text { Sum }^{-}\left(t, g, \ell s(t), f, g^{\prime}, \ell^{\prime}\right) \\
\hline
\end{array}
$$

(CFL START)

$$
\Omega(g, l s, s s) \quad \forall u \neq t . \mathcal{N}(u, g, l s(u))
$$

$P(t, g, \ell s(t), g, \ell s(t))$

dure summarization and may thus terminate in a lot of cases where the algorithm of this section might not.

\section{Model checking with summarization}

In this section, we describe our two-level model checking algorithm. The algorithm operates on the instrumented multithreaded program defined in Section 4. It also uses the partitions $\{(\mathcal{R}(t), \mathcal{L}(t), \mathcal{N}(t)) \mid t \in$ Tid $\}$ defined in Section 4. The algorithm maintains the following relations for performing summarization.

\section{Relations}

$$
\begin{aligned}
& P \subseteq \text { Tid } \times\left(\text { Global } \times \text { Local }^{\#}\right) \\
& \times\left(\text { Global } \times \text { Local }^{\#}\right) \\
& \text { Sum } \subseteq \text { Tid } \times\left(\text { Global } \times \text { Local } \text { H }^{*}\right) \\
& \times\left(\text { Global } \times \text { Local }^{\#}\right) \\
& \text { Sum }^{+} \subseteq \text { Tid } \times\left(\text { Global } \times \text { Local }{ }^{\#}\right) \\
& \times\left(\text { Global } \times \text { Local }^{\#}\right) \\
& \times \text { Frame } \\
& \text { Sum }^{-} \subseteq \text { Tid } \quad \times\left(\text { Global } \times \text { Local }^{\#}\right) \\
& \times \text { Frame } \\
& \times\left(\text { Global } \times \text { Local }^{\#}\right) \\
& \text { Mark } \subseteq \text { Tid } \times\left(\text { Global } \times \text { Local }^{\#}\right)
\end{aligned}
$$

\subsection{Algorithm}

Our model checking algorithm operates in two levels. The firstlevel reachability algorithm is similar to the algorithm in the previous section and maintains a set of reachable states $\Omega$. But it does not use $U, U^{+}$and $U^{-}$directly. Instead, it calls into a secondlevel summarization algorithm that uses $U, U^{+}$and $U^{-}$to compute four relations-P, Sum, Sum ${ }^{+}$and $\mathrm{Sum}^{-}$. Of these four relations, the last three play roles similar to $U, U^{+}$and $U^{-}$and are used to communicate results back to the first-level algorithm. Ruleset 2 gives the rules for the first-level reachability algorithm and Ruleset 3 gives the rules for the second-level summarization algorithm.
Ruleset 3: Level II-Summarization

(CFL STEP)

$$
\frac{P\left(t, g_{1}, \ell_{1}, g_{2}, \ell_{2}\right) \quad U\left(t, g_{2}, \ell_{2}, g_{3}, \ell_{3}\right) \quad \neg \mathcal{N}\left(t, g_{2}, \ell_{2}\right)}{P\left(t, g_{1}, \ell_{1}, g_{3}, \ell_{3}\right)}
$$

(CFL PUSH)

$$
\frac{P\left(t, g_{1}, \ell_{1}, g_{2}, \ell_{2}\right) \quad U^{+}\left(t, \ell_{2}, \ell_{3}, f\right) \quad \neg \mathcal{N}\left(t, g_{2}, \ell_{2}\right)}{P\left(t, g_{2}, \ell_{3}, g_{2}, \ell_{3}\right)}
$$

(CFL POP)

$$
\begin{aligned}
& P\left(t, g_{1}, \ell_{1}, g_{2}, \ell_{2}\right) \quad U^{+}\left(t, \ell_{2}, \ell_{3}, f\right) \quad \neg \mathcal{N}\left(t, g_{2}, \ell_{2}\right) \\
& \operatorname{Sum}^{-}\left(t, g_{2}, \ell_{3}, f, g_{3}, \ell_{4}\right) \\
& P\left(t, g_{1}, \ell_{1}, g_{3}, \ell_{4}\right)
\end{aligned}
$$

$\left(\mathrm{CFL} \mathrm{SUM}^{-}\right)$

$$
\frac{P\left(t, g_{1}, \ell_{1}, g_{2}, \ell_{2}\right) \quad U^{-}\left(t, \ell_{2}, f, \ell_{3}\right) \neg \mathcal{N}\left(t, g_{2}, \ell_{2}\right)}{\operatorname{Sum}^{-}\left(t, g_{1}, \ell_{1}, f, g_{2}, \ell_{3}\right)}
$$

(CFL SUM)

$$
\begin{array}{cl}
P\left(t, g_{1}, \ell_{1}, g_{2}, \ell_{2}\right) & \mathcal{N}\left(t, g_{2}, \ell_{2}\right) \\
\hline \operatorname{Sum}\left(t, g_{1}, \ell_{1}, g_{2}, \ell_{2}\right) & \operatorname{Mark}\left(t, g_{1}, \ell_{1}\right)
\end{array}
$$

$\left(\mathrm{CFL} \mathrm{SUM} M^{+}\right)$

$$
\frac{P\left(t, g_{1}, \ell_{1}, g_{2}, \ell_{2}\right) \quad U^{+}\left(t, \ell_{2}, \ell_{3}, f\right) \quad \neg \mathcal{N}\left(t, g_{2}, \ell_{2}\right)}{\operatorname{Mark}\left(t, g_{2}, \ell_{3}\right)}
$$

Let us refer to elements of $\left(\right.$ Global $\times$ Local $\left.^{\#}\right)$ as nodes. Then, the relations $P$, Sum $\mathrm{Sum}^{+}$and $\mathrm{Sum}^{-}$are all edges since they connect a pair of nodes. The relation Mark is a subset of nodes. We refer to the relations Sum, $\mathrm{Sum}^{+}$and $\mathrm{Sum}^{-}$as summary edges. These summary edges are computed by the summarization rules (Ruleset 3 ). The reachability rules and the summarization rules communicate with each other in the following way. The rule (CFL START) creates an edge in $P$ for a thread $t$ when every other thread is outside a transaction. Once summarization has been initiated via (CFL START) from the first level, it continues for as long as a transaction lasts, that is, until the condition $\mathcal{N}\left(t, g_{2}, \ell_{2}\right)$ becomes true of a target state $\left(g_{2}, \ell_{2}\right)$. The summary edges, Sum, Sum ${ }^{+}$, and $\mathrm{Sum}^{-}$, generated by summarization are used by the reachability rules to do model checking, via the rules STEP, PUSH and POP in Ruleset 2.

The edges in $P$ correspond to both "path edges" and "summary edges" in the CFL reachability algorithm for single-threaded programs [19]. The rule (CFL STEP) is used to propagate path edges within a single procedure, and the rules, (CFL PUSH) and (CFL POP) are used to propagate path edges across procedure boundaries. These rules have analogs in the CFL reachability algorithm.

Figure 6 and Figure 7 illustrate how the rules work in two situations involving function calls. In these figures we assume a fixed thread identifier $t$, and nodes of the form $(g, \ell)$ describe the global and local stores of thread $t$. A path edge $(g, \ell) \stackrel{P}{\longrightarrow}\left(g^{\prime}, \ell^{\prime}\right)$ indicates that $P\left(t, g, \ell, g^{\prime}, \ell^{\prime}\right)$ is true; at a call the edge $(g, \ell) \stackrel{U^{+}(f)}{\longrightarrow}\left(g^{\prime}, \ell^{\prime}\right)$ indicates that $U^{+}\left(t, \ell, \ell^{\prime}, f\right)$ is true, and at a return the edge $(g, \ell) \stackrel{U^{-}(f)}{\longrightarrow}\left(g^{\prime}, \ell^{\prime}\right)$ indicates that $U^{-}\left(t, \ell, f, \ell^{\prime}\right)$ is true; edges labeled with Sum, Sum $^{+}$and Sum $^{-}$are interpreted similarly. We explain how to infer some edges from other edges in the figures; the inferred edges are dashed. 
caller

callee

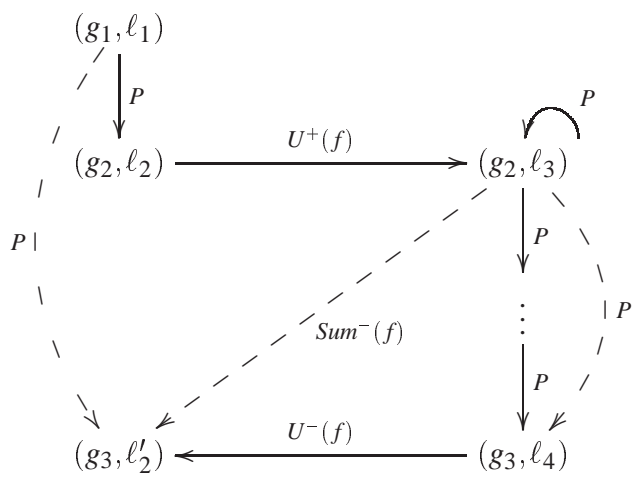

where

$-P\left(t, g_{2}, \ell_{3}, g_{2}, \ell_{3}\right)$ is inferred by (CFL PUSH)

$-P\left(t, g_{2}, \ell_{3}, g_{3}, \ell_{4}\right)$ is inferred by (CFL STEP)

$-\operatorname{Sum}^{-}\left(t, g_{2}, \ell_{3}, f, g_{3}, \ell_{2}^{\prime}\right)$ is inferred by $\left(\mathrm{CFL} \mathrm{SUM}^{-}\right)$

$-P\left(t, g_{1}, \ell_{1}, g_{3}, \ell_{2}^{\prime}\right)$ is inferred by (CFL POP)

Figure 6. Application of (CFL-POP)

In Figure 6 a caller is being summarized from state $\left(g_{1}, \ell_{1}\right)$ to the point of call at state $\left(g_{2}, \ell_{2}\right)$ by the path edge $P\left(t, g_{1}, \ell_{1}, g_{2}, \ell_{2}\right)$. At the call, indicated by the edge $U^{+}\left(t, \ell_{2}, \ell_{3}, f\right)$, a self-loop $P\left(t, g_{2}, \ell_{3}, g_{2}, \ell_{3}\right)$ on the entry state $\left(g_{2}, \ell_{3}\right)$ of the callee is inferred to start off a new procedure summary. After some computation steps in the callee a return point $\left(g_{3}, \ell_{4}\right)$ is reached, and the summary edge $\mathrm{Sum}^{-}\left(t, g_{2}, \ell_{3}, g_{3}, \ell_{2}^{\prime}\right)$ is inferred, which connects the entry state of the callee to the state immediately following the return in the caller. Finally, the path edge $P\left(t, g_{1}, \ell_{1}, g_{3}, \ell_{2}^{\prime}\right)$ is inferred to connect the original state $\left(g_{1}, \ell_{1}\right)$ to the state following the return.

It is important to note that the rules are designed to handle the complications that arise from a transaction terminating inside a function. Due to such a transaction, a summary edge may end before the return point or begin after the entry point of a callee. The rules ensure that, if a summary for a function is only partial (i.e., it does not span across both call and return), then the reachability level will execute both the call and return actions, via the PUSH and POP rules. These situations could involve scheduling other threads before, during, or after the call, as defined by the reachability relation $\Omega$.

Figure 7 illustrates inference of a partial summary for a function in which a transaction begins at the entry state $\left(\right.$ at $\left.\left(g_{2}, \ell_{3}\right)\right)$ but ends (at $\left.\left(g_{3}, \ell_{4}\right)\right)$ before a return point. The end of the transaction is indicated by the fact that $\mathcal{N}\left(t, g_{3}, \ell_{4}\right)$ is true. A partial summary up to the transaction boundary is cached in the edge $\operatorname{Sum}\left(t, g_{2}, \ell_{3}, g_{3}, \ell_{4}\right)$, which is inferred by rule (CFL SUM). Because this summary does not span across the entire call, the reachability algorithm must execute the call. This is ensured by the inference of $\operatorname{Mark}\left(g_{2}, \ell_{3}\right)$ at the same time that the fact $\operatorname{Sum}\left(t, g_{2}, \ell_{3}, g_{3}, \ell_{4}\right)$ is inferred by (CFL SUM). The fact $\operatorname{Mark}\left(g_{2}, \ell_{3}\right)$ allows the inference of $\mathrm{Sum}^{+}\left(t, g_{1}, \ell_{1}, g_{2}, \ell_{3}, f\right)$, which in turn is used by the reachability rule (PUSH) to execute the call. After executing the call, the partial summary edge $\operatorname{Sum}\left(t, g_{2}, \ell_{3}, g_{3}, \ell_{4}\right)$ is available to the reachability level, via rule (STEP).

At state $\left(g_{3}, \ell_{4}\right)$ a new transaction summary begins via (CFL caller

callee

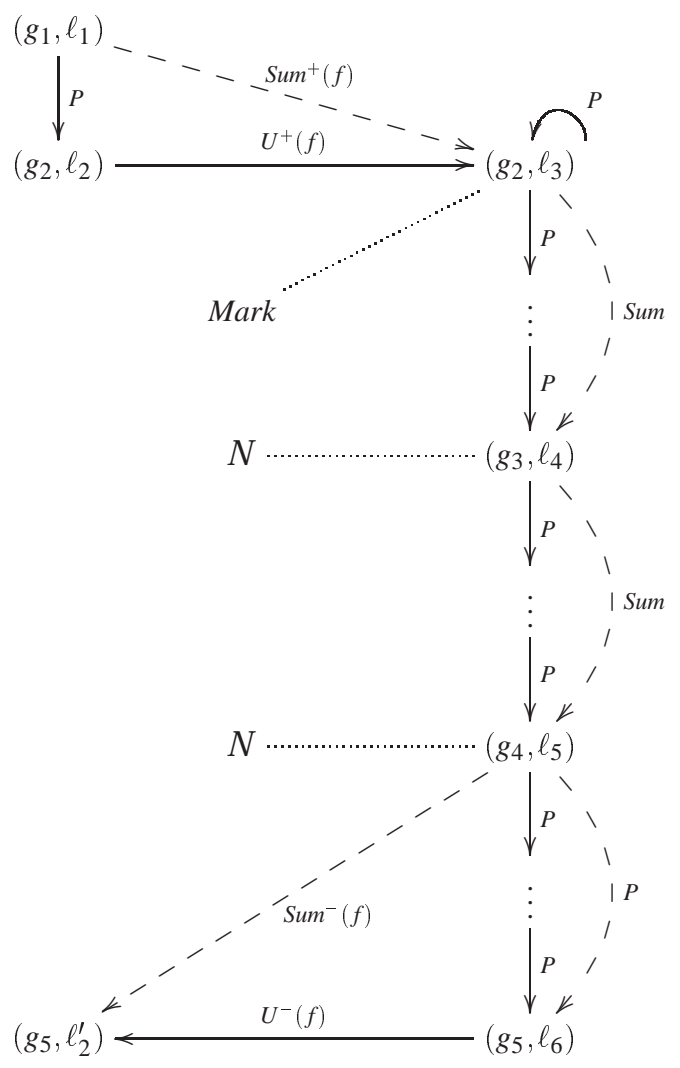

where

$-P\left(t, g_{2}, \ell_{3}, g_{2}, \ell_{3}\right)$ is inferred by (CFL PUSH)

- Sum $\left(t, g_{2}, \ell_{3}, g_{3}, \ell_{4}\right)$ is inferred by (CFL SUM)

- Mark $\left(t, g_{2}, \ell_{3}\right)$ is inferred by (CFL SUM)

- $\operatorname{Sum}^{+}\left(t, g_{1}, \ell_{1}, g_{2}, \ell_{3}, f\right)$ is inferred by $\left(\mathrm{CFL} \mathrm{SUM}^{+}\right)$

- Sum $\left(t, g_{3}, \ell_{4}, g_{4}, \ell_{5}\right)$ is inferred by (CFL SUM)

$-\operatorname{Sum}^{-}\left(t, g_{4}, \ell_{5}, f, g_{5}, \ell_{2}^{\prime}\right)$ is inferred by $\left(\mathrm{CFL} \mathrm{SUM}^{-}\right)$

Figure 7. Partial procedure summaries

STEP) (there is a self-loop $P\left(t, g_{3}, \ell_{4}, g_{3}, \ell_{4}\right)$ which is not shown for simplicity). The summary continues until the new transaction ends at $\left(g_{4}, \ell_{5}\right)$. At that point, the summary edge $\operatorname{Sum}\left(t, g_{3}, \ell_{4}, g_{4}, \ell_{5}\right)$ is inferred by rule (CFL SUM). Finally, the summary $\operatorname{Sum}^{-}\left(t, g_{4}, \ell_{5}, f, g_{5}, \ell_{2}^{\prime}\right)$ is inferred for the transition from $\left(g_{4}, \ell_{5}\right)$ across the return point at $\left(g_{5}, \ell_{6}\right)$, via rule $\left(\mathrm{CFL} \mathrm{SUM}{ }^{-}\right)$.

To summarize (!), a summary edge (either Sum, Sum ${ }^{+}$, or Sum ${ }^{-}$) is computed by the summarization algorithm under any one of the following three conditions:

- When a transaction ends at an edge $P\left(t, g_{1}, \ell_{1}, g_{2}, \ell_{2}\right)$ (indicated by $\left.\mathcal{N}\left(t, g_{2}, \ell_{2}\right)\right)$, the rule (CFL SUM) is used to generate a Sum edge. In addition, we mark the start-state of the call using $\operatorname{Mark}\left(t, g_{1}, \ell_{1}\right)$.

- Whenever a start state of a call is marked, the rule (CFL $\mathrm{SUM}^{+}$) generates a Sum ${ }^{+}$edge at every corresponding call site, and also propagates the marking to the caller. This mark- 
ing can result in additional $\mathrm{Sum}^{+}$edges being generated by iterated application of the rule ( $\left.\mathrm{CFL} \mathrm{SUM}{ }^{+}\right)$.

- When a procedure return is encountered, a $\mathrm{Sum}^{-}$edge is generated by rule (CFL SUM ${ }^{-}$).

\subsection{Correctness}

The correctness of our algorithm depends on conditions C1 and C2 from Section 4. However, since this algorithm computes the least fixpoint over a different set of equations, the condition C3 is modified to the following condition C3'. In order to state condition C3', we define the relation $P\left(t, g_{1}, \ell_{1}, g_{2}, \ell_{2}\right) \vdash \operatorname{Sum}\left(t, g_{1}, \ell_{1}, g_{3}, \ell_{3}\right)$ to hold if and only if there exists a proof tree using Ruleset 3 at whose root is an application of (CFL STEP) with $P\left(t, g_{1}, \ell_{1}, g_{2}, \ell_{2}\right)$ among its premises and at one of whose leaves is an application of (CFL SUM) with $\operatorname{Sum}\left(t, g_{1}, \ell_{1}, g_{3}, \ell_{3}\right)$ among its conclusions. We define $P\left(t, g_{1}, \ell_{1}, g_{2}, \ell_{2}\right) \vdash \operatorname{Sum}^{-}\left(t, g_{1}, \ell_{1}, f, g_{3}, \ell_{3}\right)$ and $P\left(t, g_{1}, \ell_{1}, g_{2}, \ell_{2}\right) \vdash \operatorname{Sum}^{+}\left(t, g_{1}, \ell_{1}, g_{3}, \ell_{3}, f\right)$ analogously (with applications of $\left(\mathrm{CFL} \mathrm{SUM}{ }^{-}\right)$and $\left(\mathrm{CFL} \mathrm{SUM}^{+}\right)$at the leaves, respectively).

C3'. If $P\left(t, g_{1}, \ell_{1}, g_{2}, \ell_{2}\right)$ and $\mathcal{L}\left(t, g_{2}, \ell_{2}\right)$, then one of the following conditions must hold:

1. $P\left(t, g_{1}, \ell_{1}, g_{2}, \ell_{2}\right) \vdash \operatorname{Sum}\left(t, g_{1}, \ell_{1}, g_{3}, \ell_{3}\right)$ for some $g_{3}, \ell_{3}$

2. $P\left(t, g_{1}, \ell_{1}, g_{2}, \ell_{2}\right) \vdash \operatorname{Sum}^{-}\left(t, g_{1}, \ell_{1}, f, g_{3}, \ell_{3}\right)$ for some $g_{3}, \ell_{3}, f$.

3. $P\left(t, g_{1}, \ell_{1}, g_{2}, \ell_{2}\right) \vdash \operatorname{Sum}^{+}\left(t, g_{1}, \ell_{1}, g_{3}, \ell_{3}, f\right)$ for some $g_{3}, \ell_{3}, f$.

THEOREM 2. Let $\left(g_{0}, \ell s_{0}, U, U^{+}, U^{-}\right)$be the instrumented multithreaded program. Let $\Omega$ be the least fixpoint of the rules in Rulesets 2 and 3. Let the conditions $C 1, C 2$, and C3' be satisfied. If $\left(g_{0}, l s_{0}, s s_{0}\right) \rightarrow^{*}(g, l s, s s)$ and $l s(t)=$ wrong, then there is $\left(g^{\prime}, \ell s^{\prime}, s s^{\prime}\right)$ and $p$ such that $\Omega\left(g^{\prime}, \ell s^{\prime}, s s^{\prime}\right)$ and $\ell s^{\prime}(t)=\langle$ wrong, $p\rangle$.

Proof (Sketch) The proof of this theorem depends on the following lemmas.

Lemma 1: If $\Sigma(g, \ell s, s s)$ and $\ell s(t)=\langle$ wrong, $p\rangle$, then there is $\left(g^{\prime}, \ell s^{\prime}, s s^{\prime}\right)$ such that $\Sigma\left(g^{\prime}, l s^{\prime}, s s^{\prime}\right), l s^{\prime}(t)=\langle w r o n g, p\rangle$, and $\left(g^{\prime}, \ell s^{\prime}(u)\right) \in \mathcal{N}(u)$ for all $u \in$ Tid.

Lemma 2: If $\Sigma(g, \ell s, s s)$ and $(g, \ell s(u)) \in \mathcal{N}(u)$ for all $u \in T i d$, then $\Omega(g, l s, s s)$.

Lemma 3: If $\Sigma(g, l s, s s)$ and $(g, \ell s(t)) \notin \mathcal{N}(t)$, then there are $g^{\prime}$ and $\ell^{\prime}$ such that $P\left(t, g^{\prime}, l^{\prime}, g, \ell s(t)\right)$.

Lemma 4: The condition C3' implies the condition C3.

Lemma 3 is used to prove Lemma 4. Due to Lemma 4 and the preconditions of our theorem, the preconditions of Theorem 1 are satisfied. If $\left(g_{0}, l s_{0}, s s_{0}\right) \rightarrow^{*}(g, l s, s s)$ and $l s(t)=w r o n g$, then from Theorem 1 , we get $\left(g^{\prime}, \ell s^{\prime}, s s^{\prime}\right)$ and $p$ such that $\Sigma\left(g^{\prime}, \ell s^{\prime}, s s^{\prime}\right)$ and $\ell s^{\prime}(t)=\langle$ wrong, $p\rangle$. From Lemma 1, we get $\left(g^{\prime \prime}, \ell s^{\prime \prime}, s s^{\prime \prime}\right)$ such that $\Sigma\left(g^{\prime \prime}, \ell s^{\prime \prime}, s s^{\prime \prime}\right), \ell s^{\prime \prime}(t)=\langle w r o n g, p\rangle$, and $\left(g^{\prime \prime}, \ell s^{\prime \prime}(u)\right) \in \mathcal{N}(u)$ for all $u \in$ Tid. From Lemma 2 , we get $\Omega\left(g^{\prime \prime}, \ell s^{\prime \prime}, s s^{\prime \prime}\right)$.

\subsection{Termination}

In this section, we present sufficient conditions for the least fixpoint $\Omega$ of the rules in Rulesets 2 and 3 to be finite, in which case

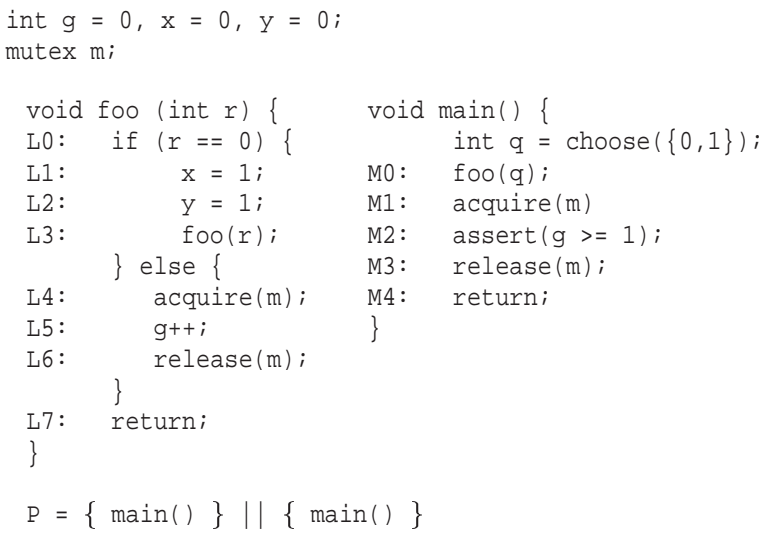

Figure 8. Nonterminating example

our summarization-based model checking algorithm will terminate. These conditions are satisfied by a variety of realistic programs that use shared variables, synchronization, and recursion.

In our notation, a frame $f \in$ Frame corresponds to a procedure call and essentially encodes the values to which the local variables (e.g., the program counter) should be set, once the procedure call returns. A frame $f$ is recursive if and only if there is a transition sequence $\left(g_{0}, \ell s_{0}, s s_{0}\right) \rightarrow^{*}(g, l s, s s)$ and a thread $t$ such that $f$ occurs more than once in the stack $s s(t)$. A frame $f$ is transactional if and only if for all transition sequences $\left(g_{0}, \ell s_{0}, s s_{0}\right) \rightarrow^{*}(g, \ell s, s s)$ and for all $t \in$ Tid, if $f$ occurs on the stack $s s(t)$, then $(g, \ell s(t)) \notin \mathcal{N}(t)$. If $f$ is transactional, then in any execution, after a thread $t$ pushes $f$ on the stack, execution continues with all states outside $\mathcal{N}(t)$ until $f$ is popped.

THEOREM 3. Suppose the domains Tid, Global, Local, and Frame are all finite. If every recursive frame $f \in$ Frame is transactional, then the set of reachable states $\Omega$ is finite, and the model checking algorithm based on Rulesets 2 and 3 terminates.

Proof (Sketch) Because the sets Global, Local, Tid and Frame are finite, it immediately follows that the relations $P$, Sum $_{\text {Sum }}{ }^{+}$, $\mathrm{Sum}^{-}$, and Mark computed by the summarization rules in Ruleset 3 are finite. Consider first the summarization rules acting on a sequence of transitions following the push of a recursive frame $f$. The rule (CFL SUM) cannot be applied to any premise of the form $P\left(t, g, \ell, g^{\prime}, \ell^{\prime}\right)$. The reason is that $f$ is transactional, and therefore $\left(g^{\prime}, \ell^{\prime}\right) \notin \mathcal{N}(t)$. Hence, no facts of the form $\operatorname{Mark}(t, g, \ell)$ or $\operatorname{Sum}\left(t, g, \ell, g^{\prime}, \ell^{\prime}\right)$ can be deduced due to any pair $\left(g^{\prime}, \ell^{\prime}\right)$. Because no such fact $\operatorname{Mark}(t, g, \ell)$ can be deduced, the rule $\left(\mathrm{CFL} \mathrm{SUM}^{+}\right)$, in turn, cannot be applied to any premise of the form $P\left(t, g, \ell, g^{\prime}, \ell^{\prime}\right)$, and hence no fact of the form $\operatorname{Sum}^{+}\left(t, g, \ell, g^{\prime}, \ell^{\prime}, f\right)$ can be deduced.

Consider next the reachability rules for the set $\Omega$ acting on a sequence of states following the push of a recursive frame $f$. By the argument above, no facts of the form $\operatorname{Sum}\left(t, g, \ell, g^{\prime}, \ell^{\prime}\right)$ and no facts of the form $\operatorname{Sum}^{+}\left(t, g, \ell, g^{\prime}, \ell^{\prime}, f\right)$ can be deduced. It follows that only the reachability rules (INIT), (STEP), and (POP) can be applied. Because none of these rules push frames on the stacks, only finitely many facts of the form $\Omega(g, l s, s s)$ can be deduced from the push of a recursive frame. Since only the set Stacks can be infinite on a program and non-recursive frames can generate only finitely many distinct stacks, it follows that only finitely many facts $\Omega(g, \ell s, s s)$ can be deduced from a program all of whose recursive frames are transactional. 
Figure 8 shows an example program on which our two-level algorithm does not terminate. Here, the procedure foo is both recursive and not transactional due to the accesses it makes to the global variables $x$ and $y$. As a result, Sum $^{+}$edges are returned by the summarization algorithm for the recursive call inside procedure foo, and consequently the reachability algorithm does not terminate.

\subsection{Single-threaded programs}

In a single threaded program, we can make the set $\mathcal{N}(1)$ for the single thread contain just the initial state of the program, and the states in which the thread has gone wrong. Suppose the program does not reach an error state. Then, the rule (CFL SUM) can never be applied and the summarization rules will never generate Sum or Sum ${ }^{+}$ edges. Consequently, the reachability rules will never explore states in which the stack is non-empty, and the model checking algorithm with summarization specializes to CFL reachability. The summary of a procedure contains only $\mathrm{Sum}^{-}$edges and is identical to the summary produced by the CFL reachability algorithm.

\section{Related work}

Recently, several papers have used the idea of reduction to develop analyses for concurrent programs. Flanagan and Qadeer developed a type system leveraging the ideas of reduction and transactions to verify atomicity in multithreaded programs $[15,14]$. Their type system was inspired by the Calvin-R static checking tool [16]. Calvin-R supports modular verification of multithreaded programs by annotating each procedure with a specification; this specification is related to the procedure implementation via an abstraction relation that combines the notions of simulation and reduction. Recently, Flanagan and Freund have also developed a dynamic atomicity checker called Atomizer for multithreaded Java programs [11]

A number of dataflow techniques have been devised to analyze programs with both concurrency and procedure calls. Duesterwald and Soffa use a system of dataflow equations to check if two statements in a concurrent program can potentially execute in parallel [7]. Their analysis is conservative and restricted to Ada rendezvous constructs. Dwyer and Clarke check properties of concurrent programs by dataflow analysis, but use inlining to flatten procedure calls [8]. Flow-insenstive analyses are independent of the ordering between program statements and can be generalized easily to multithreaded programs with procedure calls. Rinard presents a survey of techniques for analysis of concurrent programs [20].

Ramalingam proved the undecidability of assertion checking with both concurrency and procedure calls [18]. The proof is by reduction from the undecidable problem of checking the emptiness of the intersection of two context-free languages. Bouajjani, Esparza, and Touili present an analysis that constructs abstractions of context-free languages [4]. The abstractions are chosen so that the emptiness of the intersection of the abstractions is decidable. Their analysis is sound but incomplete due to overapproximation in the abstractions. In contrast, our work operates on the concrete multithreaded program and uses summaries to gain reuse, scalability, and termination in a number of cases.

Alur and Grosu have studied the interaction between concurrency and procedure calls in the context of refinement between STATECHART programs [1]. At each step of the refinement process, their system allows either the use of nesting (the equivalent of procedures) or parallelism, but not both. Also, recursively nested modes are not allowed. In contrast, we place no restrictions on how par- allelism interacts with procedure calls, and allow recursive procedures.

For restricted models of synchronization, such as fork-join synchronization, assertion checking is decidable even with both concurrency and procedure calls. Esparza and Podelski present an algorithm for this restricted class of programs [9].

Counter machines and variants of Petri nets have been used to check assertions on concurrent programs with unbounded number of threads $[6,2]$. However, these methods handle procedure calls by inlining.

\section{Conclusions}

We have presented a novel model checking algorithm to check assertions on multithreaded programs with procedure calls. Inspired by procedure summarization in sequential programs, our algorithm attempts to use summaries to obtain reuse and scalability. Our algorithm functions in two levels. The first level performs reachability analysis and maintains an explicit stack for each thread. The second level computes a summary for each procedure. Under certain conditions (stated precisely in Theorem 3), we guarantee that our two-level algorithm will terminate even in the presence of recursion and concurrency. We are currently implementing our algorithm in a new model checker called ZING being developed at Microsoft Research.

\section{References}

[1] R. Alur and R. Grosu. Modular refinement of hierarchic reactive machines. In POPL 00: Principles of Programming Languages, pages 390-402. ACM, 2000.

[2] T. Ball, S. Chaki, and S. K. Rajamani. Parameterized verification of multithreaded software libraries. In TACAS 01: Tools and Algorithms for Construction and Analysis of Systems, LNCS 2031, pages 158-173. Springer-Verlag, 2001.

[3] T. Ball and S. K. Rajamani. Bebop: A symbolic model checker for Boolean programs. In SPIN 00: SPIN Workshop, LNCS 1885, pages 113-130. Springer-Verlag, 2000.

[4] A. Bouajjani, J. Esparza, and T. Touili. A generic approach to the static analysis of concurrent programs with procedures. In POPL 03: Principles of Programming Languages, pages 62-73. ACM, 2003.

[5] M. Das, S. Lerner, and M. Seigle. ESP: Path-sensitive program verification in polynomial time. In PLDI 02: Programming Language Design and Implementation, pages 57-69. ACM, 2002.

[6] G. Delzanno, J-F. Raskin, and L. V. Begin. Towards the automated verification of multithreaded Java programs. In TACAS 02: Tools and Algorithms for Construction and Analysis of Systems, LNCS 2280, pages 173-187. Springer-Verlag, 2002.

[7] E. Duesterwald and M. L. Soffa. Concurrency analysis in the presence of procedures using a data-flow framework. In $T A V$ 91: Testing, Analysis and Verification, pages 36-48. ACM, 1991.

[8] M. Dwyer and L. Clarke. Data flow analysis for verifying properties of concurrent programs. In FSE 94: Foundations of Software Engineering, pages 62-75. ACM, 1994. 
[9] J. Esparza and A. Podelski. Efficient algorithms for pre* and post* on interprocedural parallel flow graphs. In POPL 00: Principles of Programming Languages, pages 1-11. ACM, 2000.

[10] J. Esparza and S. Schwoon. A BDD-based model checker for recursive programs. In CAV 01: Computer Aided Verficiation, LNCS 2102, pages 324-336. Springer-Verlag, 2001.

[11] C. Flanagan and S. N. Freund. Atomizer: A dynamic atomicity checker for multithreaded programs. In POPL 04: Principles of Programming Languages. ACM, 2004.

[12] C. Flanagan and S. Qadeer. Thread-modular model checking. In SPIN 03: SPIN Workshop, LNCS 2648, pages 213-225. Springer-Verlag, 2003.

[13] C. Flanagan and S. Qadeer. Transactions for software model checking. In SoftMC 03: Software Model Checking Workshop, 2003.

[14] C. Flanagan and S. Qadeer. A type and effect system for atomicity. In PLDI 03: Programming Language Design and Implementation, pages 338-349. ACM, 2003.

[15] C. Flanagan and S. Qadeer. Types for atomicity. In TLDI 03: Types in Language Design and Implementation, pages 1-12. ACM, 2003.
[16] S. N. Freund and S. Qadeer. Checking concise specifications for multithreaded software. In FTfJP 03: Formal Techniques for Java-like Programs, 2003.

[17] R. J. Lipton. Reduction: A method of proving properties of parallel programs. In Communications of the ACM, volume 18:12, pages 717-721, 1975.

[18] G. Ramalingam. Context sensitive synchronization sensitive analysis is undecidable. ACM Trans. on Programming Languages and Systems, 22:416-430, 2000.

[19] T. Reps, S. Horwitz, and M. Sagiv. Precise interprocedural dataflow analysis via graph reachability. In POPL 95: Principles of Programming Languages, pages 49-61. ACM, 1995.

[20] M. C. Rinard. Analysis of multithreaded programs. In SAS 01: Static Analysis, LNCS 2126, pages 1-19. Springer-Verlag, 2001.

[21] M. Sharir and A. Pnueli. Two approaches to interprocedural data flow analysis. In Program Flow Analysis: Theory and Applications, pages 189-233. Prentice-Hall, 1981.

[22] B. Steffen and O. Burkart. Composition, decomposition and model checking optimal of pushdown processes. Nordic Journal of Computing, 2(2):89-125, 1995. 